\title{
Effect of tualang honey against KA-induced oxidative stress and neurodegeneration in the cortex of rats
}

\author{
Nur Shafika Mohd Sairazi ${ }^{1}$, Sirajudeen K.N.S. ${ }^{1 *}$, Mohd Asnizam Asari ${ }^{2}$, Swamy Mummedy ${ }^{1}$, Mustapha Muzaimi ${ }^{3}$ \\ and Siti Amrah Sulaiman ${ }^{4}$
}

\begin{abstract}
Background: Administration of KA on rodents has resulted in seizures, behavioral changes, oxidative stress, and neuronal degeneration on selective population of neurons in the brain. The present study was undertaken to investigate the extent of neuroprotective effect conferred by Malaysian Tualang Honey (TH), an antioxidant agent, in the cerebral cortex of rats against KA-induced oxidative stress and neurodegeneration in an animal model of KAinduced excitotoxicity.

Methods: Male Sprague-Dawley rats were randomly divided into five groups: Control, KA-treated group, TH + KAtreated group, aspirin (ASP; anti-inflammatory agent) + KA-treated group and topiramate (TPM; antiepileptic agent) + KA-treated group. The animals were pretreated orally with drinking water, TH (1.0g/kg BW), ASP (7.5mg/kg BW) or TPM $(40 \mathrm{mg} / \mathrm{kg} \mathrm{BW})$, respectively, five times at $12 \mathrm{~h}$ intervals. KA $(15 \mathrm{mg} / \mathrm{kg} \mathrm{BW})$ was injected subcutaneously 30 min after last treatment to all groups except the control group (normal saline). Behavioral change was observed using an open field test (OFT) to assess the locomotor activity of the animals. Animals were sacrificed after $2 \mathrm{~h}, 24 \mathrm{~h}$ and $48 \mathrm{~h}$ of $\mathrm{KA}$ administration.
\end{abstract}

Results: KA significantly inflicted more neuronal degeneration in the piriform cortex and heightened the predilection to seizures as compared with the control animals. Pretreatment with TH reduced the KA-induced neuronal degeneration in the piriform cortex but failed to prevent the occurrence of KA-induced seizures. In the OFT, KAinduced animals showed an increased in locomotor activity and hyperactivity and these were attenuated by $\mathrm{TH}$ pretreatment. Furthermore, TH pretreatment significantly attenuated an increase of thiobarbituric acid reactive substances level and a decrease of total antioxidant status level enhanced by KA in the cerebral cortex.

Conclusion: These results suggest that pretreatment with $\mathrm{TH}$ has a therapeutic potential against KA-induced oxidative stress and neurodegeneration through its antioxidant effect.

Keywords: Tualang honey, Kainic acid, Neurodegeneration, Oxidative stress, Behavioral change, Fluoro Jade C, Cresyl violet

\footnotetext{
* Correspondence: sirajuden@usm.my; knssiraj@yahoo.com

'Department of Chemical Pathology, School of Medical Sciences, Universiti

Sains Malaysia, Health Campus, 16150 Kubang Kerian, Kota Bharu, Kelantan,

Malaysia

Full list of author information is available at the end of the article
}

(c) The Author(s). 2017 Open Access This article is distributed under the terms of the Creative Commons Attribution 4.0 International License (http://creativecommons.org/licenses/by/4.0/), which permits unrestricted use, distribution, and reproduction in any medium, provided you give appropriate credit to the original author(s) and the source, provide a link to the Creative Commons license, and indicate if changes were made. The Creative Commons Public Domain Dedication waiver (http://creativecommons.org/publicdomain/zero/1.0/) applies to the data made available in this article, unless otherwise stated. 


\section{Background}

Excitotoxicity has been considered to be a major pathological process of neuronal death in acute and chronic neurodegenerative diseases such as Alzheimer's disease, Parkinson's disease, Huntington's disease, temporal lobe epilepsy (TLE), amyotrophic lateral sclerosis and hypoxia/ischemia [1]. Kainic acid (KA), a neurotoxicant extracted from red alga, Digenea simplex, is widely used to induce seizures and to explore the mechanism of excitotoxicity and neurodegeneration. Studies on KA-induced animal experimental model have shown series of clinical manifestations and pathological changes upon KA administration on rodents such as seizures [2], behavioral changes of rodents $[3,4]$, oxidative stress $[5,6]$ and neuropathological lesions akin to those found in patients with TLE [7].

In recent decades, there is an emerging trend to search for natural resources to combat against neurodegenerative diseases. Many studies have tested or reported the protective effect of various types of natural products against KA-induced excitotoxicity models [3, 8-11]. Many of natural products possess antioxidant activity that enables them to protect against KA -induced neuronal degeneration and KA-induced seizures. In Malaysia, Tualang honey $(\mathrm{TH})$ is widely used as a nutrient supplementation and in traditional medicine. $\mathrm{TH}$ is a wild multiflora honey produced by Asian rock bees (Apis Dorsata). The honey bears its name after a tall Tualang tree (Koompassia excelsa) where Asian rock bees build their hives. Tualang tree can be found mostly in the tropical rain forest in the northern peninsular Malaysia, southern Thailand and Borneo. TH has been reported to contain many bioactive compounds which include benzoic acid, gallic acid, syringic acid, p-coumaric acid, transcinnamic acid, caffeic acid, ferulic acid, chlorogenic acid, catechin, quercetin, kaempferol, naringenin, pinobanksin3-O-propionate and pinobanksin-3-O-butyratengenin $[12,13]$. TH has also been shown to have beneficial effect at a dose of $1.0 \mathrm{~g} / \mathrm{kg}$ body weight in diabetic [14] and ovariectomized [15] rats. Studies have demonstrated that $\mathrm{TH}$ could improve spatial learning and memory performance during chronic cerebral hypoperfusion-induced neurodegeneration [16] and induce protective effect against chronic cerebral hypoperfusion-induced neurodegeneration [17]. In addition, studies have reported that $\mathrm{TH}$ consumption could deter hippocampal morphological impairment in adult male rats [18] and improve hippocampal morphological impairment in ovariectomized rats [19]. In light of these data, we hypothesized that the protective effect of $\mathrm{TH}$ could be partly mediated through ameliorating the oxidative stress, afforded by it's rich phenolic and flavanoid antioxidants.

Despite the growing body of evidence on $\mathrm{TH}$ antioxidant and anti-inflammatory actions, its neuroprotective activity in the animal model of KA-induced excitotoxicity has remained unexplored. Therefore, this study was carried out to investigate the potential neuroprotective effect and its possible mechanism of TH against the KAinduced oxidative stress and neurodegeneration on rat cerebral cortex in an animal model of KA-induced excitotoxicity. Moreover, the significance of a blockade of the cyclooxygenase $(\mathrm{COX})$ pathway and the modulatory effect on Kainate receptors (KARs) were also determined by comparing the pretreatment between $\mathrm{TH}$ and aspirin (ASP) (non-selective inhibitor of COX) and between $\mathrm{TH}$ and topiramate (TPM) (its modulatory effect on KARs) to extend our understanding of the pharmacological intervention of TH. Previous studies have suggested that ASP and TPM appeared to alleviate the neurotoxicity induced by KA $[20,21]$.

\section{Methods}

The experimental protocol was reviewed and approved by the Animal Ethic Committee of USM [USM/Animal Ethics Approval/2011/(68) (305)]. All procedures were conducted according to guidelines approved by the Animal Ethic Committee of USM. All efforts were made to minimize the number of animals used necessary to produce reliable scientific data and to lessen animal suffering. All the section of this report was written up in accordance with the Animal Research: Reporting in Vivo Experiment (ARRIVE) Guidelines for reporting animal research [22]. A completed ARRIVE guidelines checklist is included in Additional file 1.

\section{Experimental animals}

Male, Sprague-Dawley rats, aged 8 weeks were obtained from Animal Research and Service Center (ARASC), Universiti Sains Malaysia (USM) Health Campus. Animals were housed individually in polypropylene cages with soft bedding in the ARASC housing facility. They were maintained in a well-ventilated animal room at temperature of $21 \pm 2{ }^{\circ} \mathrm{C}$ under a standard animal room condition of a 12-h light/dark cycle. Animals had free access to drinking water and food pellets ad libitum. Animals were acclimatized for at least a week and they were observed closely for any abnormality to ensure that they were in good health before starting the experiment. No abnormalities were observed.

\section{Materials}

All chemicals and reagents used in this study were of analytical grade. KA and ASP were purchased from Sigma-Aldrich Co., St. Louis, MO. Tualang honey $\left(\right.$ AgroMas $\left.^{\oplus}\right)$ was supplied by Federal Agricultural Marketing Authority (FAMA), Kedah, Malaysia. TPM was purchased from Tokyo Chemical Industry Co., Ltd., Japan. Fluoro Jade C (FJC) powder was purchased from 
Histo-Chem Inc., USA. Cresyl-violet powder was purchased from Merck, Germany.

\section{Experimental design}

Male, Sprague-Dawley rats, (aged $9-11$ weeks) weighing (mean $\pm \mathrm{SD}$ ) $290 \pm 30 \mathrm{~g}$ were used in this study. Two set of animals were carried out; one set was for study 1; behavioral and biochemical studies $(N=90)$ and another set was for study 2; histology study $(N=90)$. For each set, animals were randomly divided into five major groups $(n=18 /$ group $)$ and each major group was further randomly divided into three subgroups depending on the time of sacrifice (after $2 \mathrm{~h}, 24 \mathrm{~h}$ and $48 \mathrm{~h}$ of KA administration), with a total of 15 subgroups ( $n=6 /$ subgroup). All treatments were conducted at the day session (between 8 am and $10 \mathrm{am}$ ) and at the night session (between $8 \mathrm{pm}$ and $10 \mathrm{pm}$ ) for each treatment day.

i. Group 1: The saline-treated group (control group) Animals were treated orally with drinking water five times every $12 \mathrm{~h}$.

ii. Group 2: The KA only-treated group - Animals were treated orally with drinking water five times every $12 \mathrm{~h}$.

iii. Group 3: The TH + KA-treated group -Animals were treated orally with $\mathrm{TH}(1.0 \mathrm{~g} / \mathrm{kg}$ body weight) five times every $12 \mathrm{~h}$. The $\mathrm{TH}$ dose of $1.0 \mathrm{~g} / \mathrm{kg}$ body weight was chosen as it was previously shown to have beneficial effect in diabetic [14] and ovariectomized [15] rats.

iv. Group 4: The TPM + KA-treated group - Animals were treated orally with TPM ( $40 \mathrm{mg} / \mathrm{kg}$ body weight) five times every $12 \mathrm{~h}$. The dosage was chosen based on other studies using topiramate [23-25].

v. Group 5: The ASP + KA-treated group - Animals were treated orally with ASP $(7.5 \mathrm{mg} / \mathrm{kg}$ body weight) five times every $12 \mathrm{~h}$. The dosage was chosen based on Kim et al. study [20].

\section{Kainic acid administration}

Thirty minutes after the last administration of drinking water or honey or drugs respectively, KA $(15 \mathrm{mg} / \mathrm{kg}$ body weight; $10 \mathrm{mg} / \mathrm{ml}$ in normal saline) was administered subcutaneously (s.c.) to all groups except for the control group, which received subcutaneous injection of normal saline. The dose of KA $(15 \mathrm{mg} / \mathrm{kg}$ body weight; s.c.) has been previously reported to be sufficient to inflict a significant injury in the different regions of the brain (i.e. amygdala, hippocampus and cortex) and all tested parameters (i.e. citrulline level, total adenine nucleotides and total creatine compounds), to reach their peaks after $2 \mathrm{~h}$ following KA administration [26].

\section{Seizure characteristics}

Following KA administration, animals were monitored for $4 \mathrm{~h}$ and the time to the onset of first generalized tonic-clonic seizures (characterized by stage 4 described by Zhang et al. [2]) was recorded. Since this study used single high dose KA treatments which were associated with a high mortality rate, special efforts were made to improve the survival rate of KA-treated animals. To reduce mortality [27], diazepam $(10 \mathrm{mg} / \mathrm{kg}$ body weight; Atlantic Laboratories Corp. Ltd., Thailand) was administered intraperitoneally, approximately $90 \mathrm{~min}$ after the first generalized seizure to animals of $24 \mathrm{~h}$ and $48 \mathrm{~h}$ subgroup, except for the control groups, which received an equivalent amount of normal saline. Subcutaneous normal saline injection was administered periodically during the first $24 \mathrm{~h}$ recovery period.

\section{The open-field test procedure}

For the study 1 only, behavioral change was observed using an open field test (OFT) to assess the locomotor activity of animals. With the exception of $2 \mathrm{~h}$ subgroups, locomotor activity of animals was documented in the $24 \mathrm{~h}$ and $48 \mathrm{~h}$ subgroups from each major group. The animals were tested one at a time and handled by their tails at all times. The animals were individually placed at the central square of the OFT apparatus $(90 \mathrm{~cm} \times 90 \mathrm{~cm} \times 27 \mathrm{~cm}$, consisting of 25 equally sized squares measuring $18 \mathrm{~cm} \times 18 \mathrm{~cm}$ each). Animals were allowed to explore freely the area for $15 \mathrm{~min}$ while the locomotor activity was recorded using the video-tracking system. After each trial, the animals were returned to their cage and the apparatus was cleaned between each rat using 30\% ethyl alcohol and allowed to dry between trials. All tests were conducted between 8 am and $2 \mathrm{pm}$. The OFT was performed using an in-house built-in OFT apparatus from the Department of Neurosciences, School of Medical Sciences Universiti Sains Malaysia, Kelantan, Malaysia and SMART software (Panlab, Harvard) from BRAINetwork, Universiti Sains Malaysia, Kelantan, Malaysia. Locomotor activity of animals was assessed based on the number of line crossing (the frequency of crossing one of the black grid lines with all four paws) in the OFT apparatus in 15 min duration. The behavioral of animals were observed and analyzed by an observer blinded with respect to the experimental groups.

\section{Preparation of tissue samples}

For the study 1, depending on the time of sacrifice (after $2 \mathrm{~h}, 24 \mathrm{~h}$ and $48 \mathrm{~h}$ of KA administration), following the completion of the behavioral experiment, all major group of rats were decapitated using guillotine (Harvard Apparatus, USA) after $2 \mathrm{~h}, 24 \mathrm{~h}$ and $48 \mathrm{~h}$ of KA administration with the respective control groups. The cerebral 
cortex was rapidly, carefully removed, weighed and thoroughly washed with an ice-cold $\left(0^{\circ}-4{ }^{\circ} \mathrm{C}\right)$ normal saline to remove any excess blood. The cerebral cortex was minced to small pieces and homogenized to make 7.5\% $(\mathrm{w} / \mathrm{v})$ homogenates in ice-cold $0.1 \mathrm{M}$ sodium phosphate buffer, Ph 7.4, using ice-chilled glass-homogenizing vessel in a tissue homogenizer fitted with Teflon pestle (Glas-Col, USA) at $900 \mathrm{rpm}$ for $90 \mathrm{~s}$. Then, the homogenates were centrifuged in a refrigerated centrifuge at $4000 \mathrm{rpm}$ for $15 \mathrm{~min}$ at $4{ }^{\circ} \mathrm{C}$ to remove nuclei and debris. The resulting supernatant fraction of homogenates was used for the measurement of lipid peroxidation and total antioxidant status.

For the study 2, depending on the time of sacrifice ( $2 \mathrm{~h}, 24 \mathrm{~h}$ and $48 \mathrm{~h}$ of $\mathrm{KA}$ administration), all major groups of rats were deeply anesthetized with a mixture of ketamine/xylazine $(90 \mathrm{mg} / \mathrm{kg}$ body weight ketamine and $5 \mathrm{mg} / \mathrm{kg}$ body weight xylazine), via intramuscular injection. The toe pinch-response method was used to determine the depth of anesthesia and animals must be unresponsive before commencing perfusion process. The anaesthetized rat was transcardially perfused with ice-cold phosphate buffer saline, $\mathrm{pH} 7.4$ and followed by ice-cold $4 \%$ paraformaldehyde in $0.2 \mathrm{M}$ sodium phosphate buffer, $\mathrm{pH} 7.4$ after $2 \mathrm{~h}$, $24 \mathrm{~h}$ and $48 \mathrm{~h}$ of KA administration with the respective control groups. Brains were rapidly removed from skulls. The cerebrum was dissected and separated from the rest of the brain and then immersed in the same fixative solution at $4{ }^{\circ} \mathrm{C}$ for overnight. After post-fixation, the cerebrum was processed, embedded in paraffin and sectioned into serial 5 - $\mu$ m-thick coronal sections, taken from $4.60 \mathrm{~mm}$ and $3.60 \mathrm{~mm}$ with respect to a relative distance from Bregma [28]. Prior to histochemical staining, the sections were mounted onto poly-L-lysine coated slides and were air dried for overnight. The slides were stained with cresylviolet stain and Fluoro Jade $\mathrm{C}$ (FJC) to examine degenerating neurons.

\section{Determination of thiobarbituric acid reactive substances and total antioxidant status level}

The measurement of lipid peroxidation was determined by estimating Thiobarbituric Acid Reactive Substances (TBARS), according to the method previously described by Ohkawa et al. [29] with some modifications. TBARS level (lipid peroxidation) was expressed as nanomoles of Malondialdehyde equivalent per $g$ wet tissue (nanomoles/g wet tissue). Meanwhile, total antioxidant status (TAS) of the homogenate sample was determined as previously described by Koracevic et al. [30]. TAS level was expressed as nanomoles of uric acid equivalent per $\mathrm{g}$ wet tissue (nanomoles/g wet tissue).
Evaluation of viable cells using cresyl violet staining

Cresyl violet staining was performed to provide the estimation of neuronal loss. Cresyl violet staining stock solution was prepared by dissolving $0.1 \mathrm{~g}$ cresyl violet acetate powder (Merck, Germany) in $75 \mathrm{ml}$ of distilled water. Then, $6 \mathrm{ml}$ of cresyl violet stock solution was diluted with $50 \mathrm{ml}$ of $0.1 \mathrm{M}$ acetate buffer ( $\mathrm{pH}$ 3.6) to make cresyl violet working solution.

In brief, slides were treated sequentially with the following steps. First, the sections were deparaffinized with xylene and a series of descending grade of ethanol $(100 \%, 95 \%$ and $80 \%)$ and then brought to water. Following tissue hydration, the sections were stained with cresyl violet and then rinsed with distilled water. The sections were air dried overnight, cleared in xylene and then cover slipped with Dibutylphthalate Polystyrene Xylene (DPX) mounting media (Sigma, USA).

The cresyl violet-stained sections were visualized and imaged using Olympus BX41 microscope equipped with a high resolution digital camera system and a desktop computer preinstalled with image analysis software, analySIS FIVE. Viable cells were counted and defined as cells with normal morphology, exhibiting round nuclei stained with cresyl violet-stained.

\section{Evaluation of degenerating neurons using Fluoro Jade $C$ staining}

In order to further evaluate neuronal degeneration in the cortex of rats, FJC histochemical staining was performed using the method described by Schmued et al. [31]. FJC is an anionic fluorescent dye, a specific marker of degenerating neurons. FJC staining stock solution was prepared by dissolving $10 \mathrm{mg}$ of dry powder FJC (HistoChem Inc., USA) in $100 \mathrm{ml}$ distilled water. Then, $10 \mathrm{ml}$ of stock solution was added to $90 \mathrm{ml}$ of $0.1 \%$ acetic acid, producing $0.001 \%$ concentration of FJC working solution.

In brief, slides were then treated sequentially with the following steps: sections were deparaffinized with xylene and a series of descending graded series of ethanol $(100 \%, 95 \%$ and $80 \%)$ and then immersed in distilled water. After tissue hydration, slides were immersed in $0.1 \%$ potassium permanganate solution for $20 \mathrm{~min}$ with slow shaking in the dark and then rinsed with distilled water for $2 \mathrm{~min}$. Slides were incubated in $0.001 \%$ FJC in $0.1 \%$ acetic acid for $10 \mathrm{~min}$ with slow shaking in the dark. Then, slides were rinsed 3 times with distilled water. The sections were air dried overnight, cleared in xylene and then cover slipped with DPX mounting media (Sigma, USA).

The FJC-labelled sections were visualized and imaged using an Olympus BX41 fluorescence microscope using a filter system designed for visualizing fluorescein (Excitation peak:4 nm, Emission peak: $525 \mathrm{~nm}$ ) 
equipped with a high resolution digital camera system and desktop computer preinstalled with image analysis software, analySIS FIVE. Fluoro-jade-positive cell were counted and defined as a bright green fluorescence, while normal neurons appeared even darker than background with lightly staining nucleoli or were unstained.

\section{Neuronal quantification}

Three representative sections of the cerebrum were selected for each animal that were used to quantify neuronal degeneration. These sections were taken from Bregma $4.60 \mathrm{~mm}$ to Bregma $3.60 \mathrm{~mm}$ [28] at a regular space interval of $100 \mu \mathrm{m}$ with $5 \mu \mathrm{m}$ thick for each rat from each group. Using analySIS FIVE image analysis software, five non-overlapping areas were identified on piriform cortex and photographed at 200x magnification. The data of these images were saved into computer. Three of these images were randomly selected for neuronal quantification. Neuronal cell counts were performed using Image J image analysis software (National Institute of Health, USA) and the data were then averaged for each animal. The neuronal quantification was performed by an observer blinded with respect to the experimental groups.

\section{Statistical analysis}

All data were analyzed using SPSS software version 22 (SPSS Statistics IBM, Chicago, USA). Statistical significance of differences was determined by Kruskal-Wallis followed by Mann-Whitney U (MW) test. Data was considered statistically significant when $\mathrm{p}$ value was less than $0.05(p<0.05)$. Results are expressed as median (Interquartile Range; IqR).

\section{Results}

\section{Administration of KA induced seizures}

Administration of KA (15 mg/kg body weight, s.c.) induced epileptic seizures in all KA-treated rats and KAtreated groups that received pretreatment of TH, ASP and TPM. No seizure behavior was observed in the control group. The onset of seizure occurred most frequently within $1 \mathrm{~h}$ to $4 \mathrm{~h}$ post-KA administration. All KA-treated rats exhibited progressive motor seizures. It started with staring spells in which rats seemed to be in a motion arrest. This was followed by wet dog shakes which progressively became frequent in nature. Then, the animals displayed hyperactive behavior in which they had repeated head nodding with the intervals of continuous walking and chewing. After that, rats started to rear up on their hind limbs and then developed frequent and prolonged rearing, accompanied by forelimb clonic jerks, salivation and white foaming at the mouth (first generalized seizure). Next, the rats were falling or had loss of balance while rearing. These rearing and falling episodes continued until the rats were injected with diazepam $(10 \mathrm{mg} / \mathrm{kg}$ body weight) approximately $90 \mathrm{~min}$ following the onset of the first generalized seizure. The rats in the control group displayed normal behavior such as walking, sniffing, grooming and exploring.

Statistical analysis indicated that there was no significant difference $(p>0.05)$ in the time to the onset of the first generalized seizures between groups, $\left[x^{2}(3)=4.72\right.$, $(N=48), p=0.1937$ ] (Table 1$)$. The median values of the time to the onset of the first generalized seizures in the KA-induced rats pre-treated with TH, ASP and TPM were no significantly different $(p>0.05)$ from those of the KA-induced rats. Therefore, pretreatment with $\mathrm{TH}$, ASP and TPM did not have any apparent anticonvulsant effect in KA-induced seizures.

\section{Effect of KA-induced excitotoxicity on the number of line} crossing in the OFT

Statistical analysis of number of line crossing in the OFT indicated that there was a significant difference $(p<0.05)$ in the number of line crossing between the groups for all $24 \mathrm{~h}$ and $48 \mathrm{~h}$ subgroups of KA administration, as shown in Table $2\left[24 \mathrm{~h}\right.$ subgroups $=\mathrm{x}^{2}$ (4) $=19.79,(N=30), p=0.0005 ; 48 \mathrm{~h}$ subgroups $=\chi^{2}$ $(4)=11.71, \quad(N=30), p=0.0196]$. Post hoc test revealed there was a significant difference $(p<0.05)$ in the number of line crossing in the OFT between the control and KA-treated groups in $24 \mathrm{~h}$ and $48 \mathrm{~h}$ subgroups, and between the control and KA-induced groups pre-treated with TPM for $24 \mathrm{~h}$ subgroups of KA administration. In the OFT, KA-treated group was increased in the locomotor activity (based on number of line crossing) and hyperactivity.

There was also a significant difference $(p<0.05)$ in the number of line crossing in the OFT between KA-treated groups and KA-induced groups pre-treated with $\mathrm{TH}$ of $24 \mathrm{~h}$ and $48 \mathrm{~h}$ of KA administration (Table 2). This indicated that the increased locomotor activity induced by KA was attenuated by $\mathrm{TH}$ pre-treatment. There was a significant difference $(p<0.05)$ in the number of line

Table 1 Effect of KA-induced excitotoxicity on the time to the onset of the first generalized seizures between groups

\begin{tabular}{llll}
\hline Group & \begin{tabular}{l} 
Onset time of the first \\
generalized seizures $(\mathrm{min})$ \\
\cline { 4 - 4 }
\end{tabular} & \multicolumn{2}{l}{ Kruskal-Wallis test $(N=48)$} \\
\cline { 3 - 4 } KA & $76.50(38.50)$ & $4.72(3)$ & 0.1937 \\
TH + KA & $65.00(23.50)$ & & \\
TPM + KA & $64.50(23.50)$ & & \\
ASP + KA & $62.50(13.25)$ & & \\
\hline
\end{tabular}

The results were expressed as the median (IqR). The significant difference was determined by non-parametric test, Kruskal-Wallis test. Mann-Whitney $\mathrm{U}$ test was not performed because there was no significant difference $(p>0.05)$ in the median values with Kruskal-Wallis test 
Table 2 Effect of KA-induced excitotoxicity on the number of line crossing in the OFT

\begin{tabular}{|c|c|c|c|c|c|c|c|}
\hline \multirow[t]{2}{*}{ Subgroups } & \multicolumn{5}{|c|}{$\begin{array}{l}\text { Number of line crossing } \\
\text { Median (lqR) }(n=6)\end{array}$} & \multicolumn{2}{|c|}{ Kruskal-Wallis test $(N=30)$} \\
\hline & CONTROL & $\mathrm{KA}$ & $\mathrm{TH}+\mathrm{KA}$ & $\mathrm{TPM}+\mathrm{KA}$ & $\mathrm{ASP}+\mathrm{KA}$ & $x^{2}$ stat $(\mathrm{df})$ & $p$ value \\
\hline $24 \mathrm{H}$ & $190.00(57.00)$ & $259.50(50.50)^{a}$ & $160.50(45.50)^{b}$ & $101.00(57.75)^{a, b}$ & $120.00(170.25)^{b}$ & $19.79(4)$ & 0.0005 \\
\hline $48 \mathrm{H}$ & $143.00(41.75)$ & $197.50(21.25)^{a}$ & $135.00(20.75)^{b}$ & $169.00(153.25)$ & $123.50(82.00)^{b}$ & $11.71(4)$ & 0.0196 \\
\hline
\end{tabular}

The results were expressed as the median (IqR). The significant difference was determined by non-parametric test, Kruskal-Wallis test followed by Mann-Whitney $\mathrm{U}$ post-hoc test with $p<0.05$ indicates statistically significant difference. ${ }^{a} p<0.05$ versus control group (MW); ${ }^{b} p<0.05$ versus KA group (MW)

crossing in the OFT between KA-treated groups and KA-induced groups pre-treated with TPM for $24 \mathrm{~h}$ subgroups of KA administration, and between KA-treated groups and KA-induced groups pre-treated with ASP of $24 \mathrm{~h}$ and $48 \mathrm{~h}$ of $\mathrm{KA}$ administration.

\section{Effect of KA-induced excitotoxicity on TBARS level in the cerebral cortex}

Statistical analysis of the TBARS level in the cerebral cortex indicated that there was a significant difference $(p<0.05)$ in the TBARS level between the groups for all $2 \mathrm{~h}, 24 \mathrm{~h}$ and $48 \mathrm{~h}$ subgroups of KA administration, as shown in Table 3 [ $2 \mathrm{~h}$ subgroups $=\chi^{2}(4)=22.63$, $(N=30), p=0.0002 ; 24 \mathrm{~h}$ subgroups $=\chi^{2}(4)=24.18$, $(N=30), \quad p=0.0001 ; 48 \mathrm{~h}$ subgroups $=\chi^{2} \quad(4)=22.97$, $(N=30), p=0.0001]$.

Post hoc test showed that there was a significant difference $(p<0.05)$ in the TBARS level between the control and all the treatment groups that received KA, for all $2 \mathrm{~h}, 24 \mathrm{~h}$ and $48 \mathrm{~h}$ subgroups of KA administration, except for between control groups and KA-induced groups pre-treated with TH and TPM after $2 \mathrm{~h}$ of KA administration. The analysis indicated that the TBARS level in KA- treated, $\mathrm{TH}+\mathrm{KA}$ treated, $\mathrm{TPM}+\mathrm{KA}$ treated and ASP + KA treated groups were significantly higher as compared with relative control groups after $2 \mathrm{~h}, 24 \mathrm{~h}$ and $48 \mathrm{~h}$ following KA administration, respectively (Table 3 ).

There was also a significant difference $(p<0.05)$ in the TBARS level between KA-treated groups and KAinduced groups pre-treated with $\mathrm{TH}$ after $2 \mathrm{~h}, 24 \mathrm{~h}$ and $48 \mathrm{~h}$ of KA administration (Table 3 ). This showed that
TH pre-treatment significantly attenuated an increase of TBARS level induced by KA. There was also a significant difference $(p<0.05)$ in the TBARS level between KA-treated groups and KA-induced groups pre-treated with TPM and ASP after $2 \mathrm{~h}, 24 \mathrm{~h}$ and $48 \mathrm{~h}$ of KA administration (Table 3 ). In addition, there was a significant difference in the TBARS level between KA-induced groups pre-treated with $\mathrm{TH}$ and KA-induced groups pre-treated with ASP after 24 h of KA administration.

\section{Effect of KA-induced excitotoxicity on TAS level in the cerebral cortex}

Statistical analysis of the TAS level in the cerebral cortex indicated that there was a significant difference $(p<0.05)$ in the TAS level between the groups for all $2 \mathrm{~h}, 24 \mathrm{~h}$ and $48 \mathrm{~h}$ subgroups of KA administration, as shown in Table 4 [ $2 \mathrm{~h}$ subgroups $=\chi^{2}(4)=25.13$, $(N=30), \quad p=0.0000 ; 24 \mathrm{~h}$ subgroups $=\chi^{2}(4)=23.69$, $(N=30), \quad p=0.0001 ; 48 \mathrm{~h}$ subgroups $=\chi^{2}(4)=25.36$, $(N=30), p=0.0000]$.

Post hoc test indicated that there was a significant difference $(p<0.05)$ in the TAS level between the control and all the treatment groups that received $\mathrm{KA}$, for all $2 \mathrm{~h}, 24 \mathrm{~h}$ and $48 \mathrm{~h}$ subgroups of KA administration. The results of analysis also showed that the TAS level in KA- treated, $\mathrm{TH}+\mathrm{KA}$ treated, TPM + KA treated and ASP + KA treated groups were significantly lowered as compared with relative control groups after $2 \mathrm{~h}, 24 \mathrm{~h}$ and $48 \mathrm{~h}$ following KA administration, respectively (Table 4 ).

There was also a significant difference $(p<0.05)$ in the TAS level between KA-treated groups and KA-induced

Table 3 Effect of KA-induced excitotoxicity on TBARS level in the cerebral cortex

\begin{tabular}{|c|c|c|c|c|c|c|c|}
\hline \multirow[t]{2}{*}{ Subgroups } & \multicolumn{5}{|c|}{$\begin{array}{l}\text { TBARS (nanomoles/g wet tissue) } \\
\text { Median (lqR) }(n=6)\end{array}$} & \multicolumn{2}{|c|}{ Kruskal-Wallis test $(N=30)$} \\
\hline & CONTROL & $\mathrm{KA}$ & $\mathrm{TH}+\mathrm{KA}$ & $\mathrm{TPM}+\mathrm{KA}$ & $\mathrm{ASP}+\mathrm{KA}$ & $x^{2}$ stat $(d f)$ & $p$ value \\
\hline $2 \mathrm{H}$ & $11.95(2.90)$ & $21.93(1.78)^{a}$ & $15.40(3.72)^{b}$ & $15.35(4.08)^{b}$ & $18.35(1.31)^{\mathrm{a}, \mathrm{b}}$ & $22.63(4)$ & 0.0002 \\
\hline $24 \mathrm{H}$ & $13.54(3.38)$ & $101.55(11.48)^{a}$ & $75.70(8.25)^{a, b}$ & $80.19(9.17)^{\mathrm{a}, \mathrm{b}}$ & $82.38(6.69)^{a, b, c}$ & $24.18(4)$ & 0.0001 \\
\hline $48 \mathrm{H}$ & $12.55(2.21)$ & $81.39(10.87)^{a}$ & $54.21(9.32)^{a, b}$ & $57.64(5.14)^{a, b}$ & $56.46(8.44)^{a, b}$ & $22.97(4)$ & 0.0001 \\
\hline
\end{tabular}

The results were expressed as the median (lqR). The significant difference was determined by non-parametric test, Kruskal-Wallis test followed by Mann-Whitney $U$ post-hoc test with $p<0.05$ indicates statistically significant difference. ${ }^{\mathrm{a}} p<0.05$ versus control group (MW); ${ }^{\mathrm{b}} p<0.05$ versus KA group (MW); ${ }^{\mathrm{c}} p<0.05$ versus $\mathrm{KA}+\mathrm{TH}$ group (MW) 
Table 4 Effect of KA-induced excitotoxicity on TAS level in the cerebral cortex

\begin{tabular}{|c|c|c|c|c|c|c|c|}
\hline \multirow[t]{2}{*}{ Subgroups } & \multicolumn{5}{|c|}{$\begin{array}{l}\text { TAS (nanomoles/g wet tissue) } \\
\text { Median (lqR) }(n=6)\end{array}$} & \multicolumn{2}{|c|}{ Kruskal-Wallis test $(N=30)$} \\
\hline & CONTROL & KA & $\mathrm{TH}+\mathrm{KA}$ & $T P M+K A$ & $A S P+K A$ & $x^{2}$ stat $(d f)$ & $p$ value \\
\hline $2 \mathrm{H}$ & $789.62(31.05)$ & $595.83(45.37)^{a}$ & $668.80(17.05)^{a, b}$ & $643.11(21.43)^{a, b, c}$ & $633.84(27.71)^{\mathrm{a}, \mathrm{b}, \mathrm{c}}$ & $25.13(4)$ & 0.0000 \\
\hline $24 \mathrm{H}$ & $798.16(17.27)$ & $533.40(18.52)^{a}$ & $631.66(29.97)^{\mathrm{a}, \mathrm{b}}$ & $643.32(54.40)^{a, b}$ & $601.23(33.85)^{a, b, c}$ & $23.69(4)$ & 0.0001 \\
\hline $48 \mathrm{H}$ & $797.59(26.24)$ & $578.87(8.92)^{a}$ & $657.57(34.49)^{a, b}$ & $664.97(41.12)^{a, b}$ & $622.71(20.48)^{a, b, c}$ & $25.36(4)$ & 0.0000 \\
\hline
\end{tabular}

The results were expressed as the median (IqR). The significant difference was determined by non-parametric test, Kruskal-Wallis test followed by Mann-Whitney $\mathrm{U}$ post-hoc test with $p<0.05$ indicates statistically significant difference. ${ }^{a} p<0.05$ versus control group (MW); ${ }^{b} p<0.05$ versus KA group (MW); ${ }^{c} p<0.05$ versus KA $+\mathrm{TH}$ group (MW)

groups pre-treated with $\mathrm{TH}$ after $2 \mathrm{~h}, 24 \mathrm{~h}$ and $48 \mathrm{~h}$ of KA administration (Table 4). Therefore, TH pretreatment significantly attenuated a decrease of in TAS level induced by KA.

There was also a significant difference $(p<0.05)$ in the TAS level between KA-treated groups and KA-induced groups pre-treated with ASP and TPM, respectively, after $2 \mathrm{~h}, 24 \mathrm{~h}$ and $48 \mathrm{~h}$ of KA administration (Table 4). In addition, there was a significant difference $(p<0.05)$ in the TAS level between KA-induced groups pretreated with $\mathrm{TH}$ and $\mathrm{KA}$-induced groups pre-treated with ASP of $2 \mathrm{~h}, 24 \mathrm{~h}$, and $48 \mathrm{~h}$ of KA administration and between KA-induced groups pre-treated with $\mathrm{TH}$ and KA-induced groups pre-treated with TPM of $2 \mathrm{~h}$ of KA administration (Table 4). This indicated that pretreatment with $\mathrm{TH}$ showed a more pronounced protective effect compared with the ASP + KA-treated treated groups.

\section{Effect of KA-induced excitotoxicity on the number of viable cells in the piriform cortex}

To evaluate the protective effect of TH, ASP and TPM against KA-induced neuronal death, we performed cresyl violet staining to quantify the number of viable cells in the piriform cortex. Systemic administration of KA caused neuronal death in the piriform cortex, as recognized by pyknotic appearance and subsequently has less viable cells, in comparison with the control groups, which have more viable cells in the piriform cortex, as shown in Fig. 1(a) -(e), Fig. 2(a) - (e), and Fig. 3(a) -(e). Viable cells were defined as cells with normal morphology, exhibiting round nuclei stained with cresyl violet.

Statistical analysis of the number of viable cells in the piriform cortex indicated that there was a a significant difference $(p<0.05)$ in the number of viable cells Table 5 [2 h subgroups $=\chi^{2}(4)=24.46,(N=30), p=0.0001 ; 24 \mathrm{~h}$ subgroups $=\chi^{2} \quad(4)=25.96, \quad(N=30), \quad p=0.0000 ; 48 \mathrm{~h}$

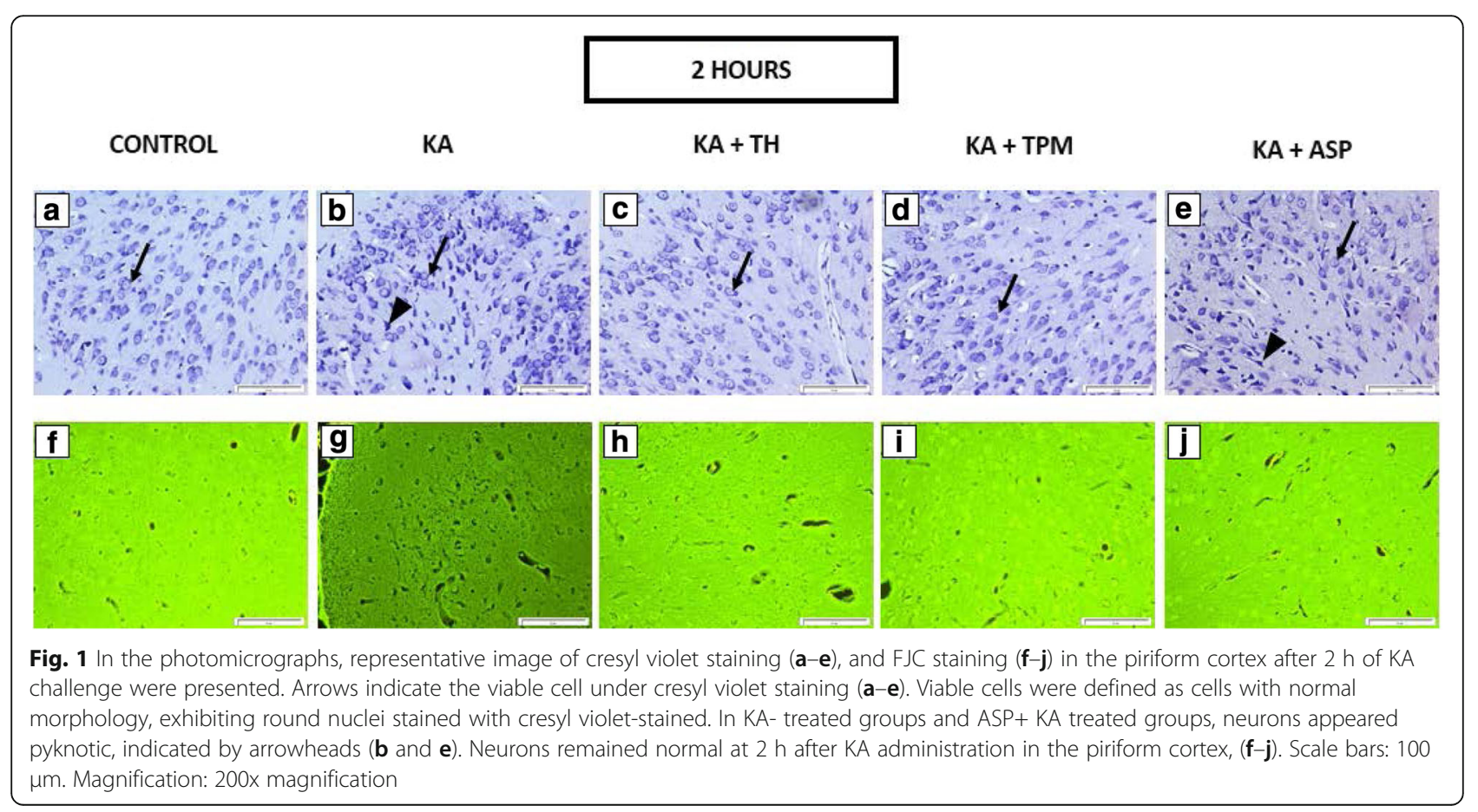




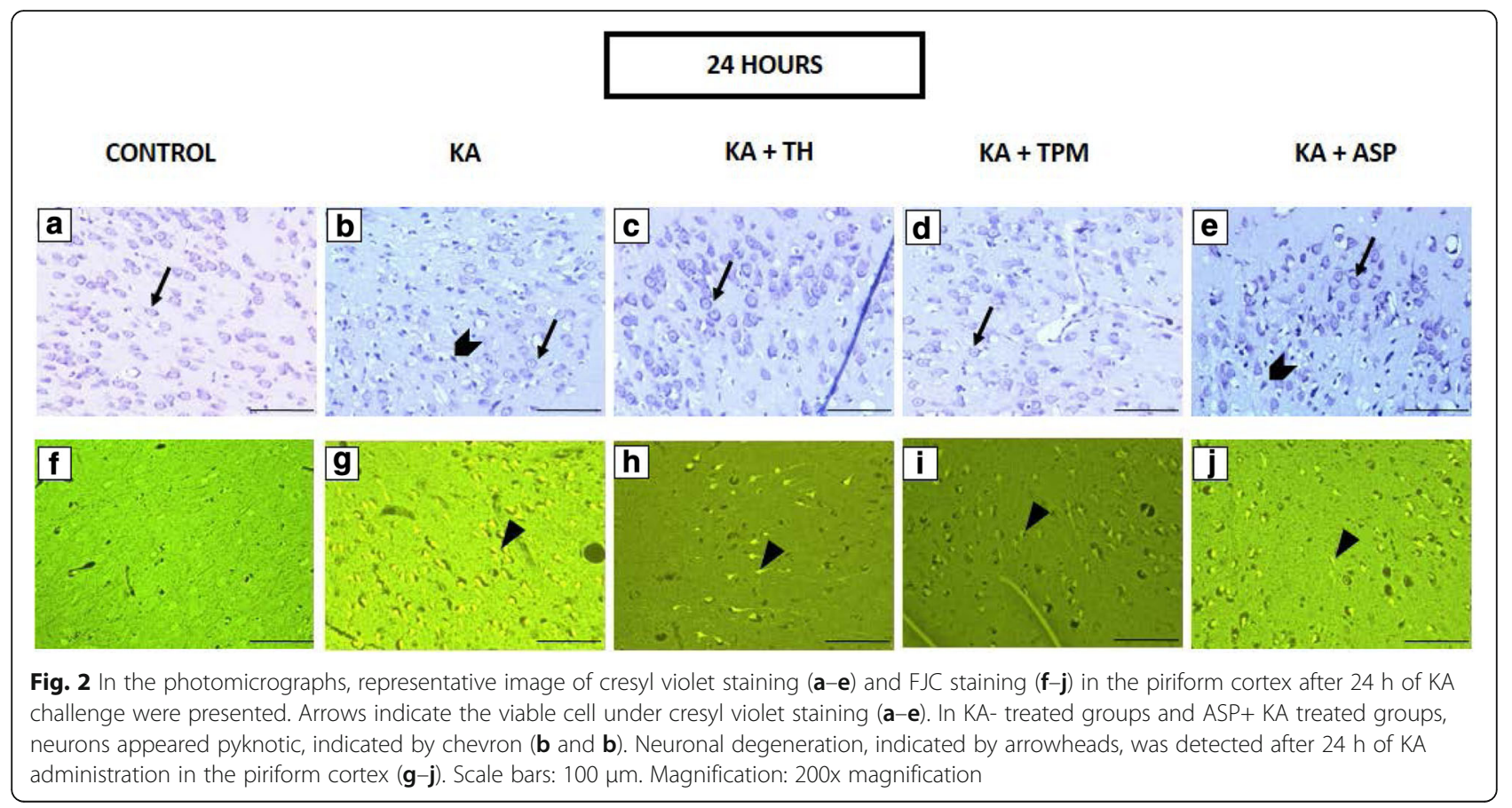

subgroups $\left.=\chi^{2} \quad(4)=24.58, \quad(N=30), \quad p=0.0001\right] . \quad$ Post hoc test revealed that there was a significant difference $(p<0.05)$ in the number of viable cells between the control and all the treatment groups that received KA, for all $2 \mathrm{~h}, 24 \mathrm{~h}$ and $48 \mathrm{~h}$ subgroups of KA administration (Table 5). This indicated that there was less viable cells in $\mathrm{KA}$ - treated, $\mathrm{TH}+\mathrm{KA}$ treated, $\mathrm{TPM}+\mathrm{KA}$ treated and
ASP + KA treated groups and they were significantly reduced as compared with the relative control groups after $2 \mathrm{~h}, 24 \mathrm{~h}$ and $48 \mathrm{~h}$ following KA administration, respectively (Table 5).

There was also a significant difference $(p<0.05)$ in the number of viable cells between KA-treated groups and KA-induced groups pre-treated with $\mathrm{TH}$ after $2 \mathrm{~h}$ and

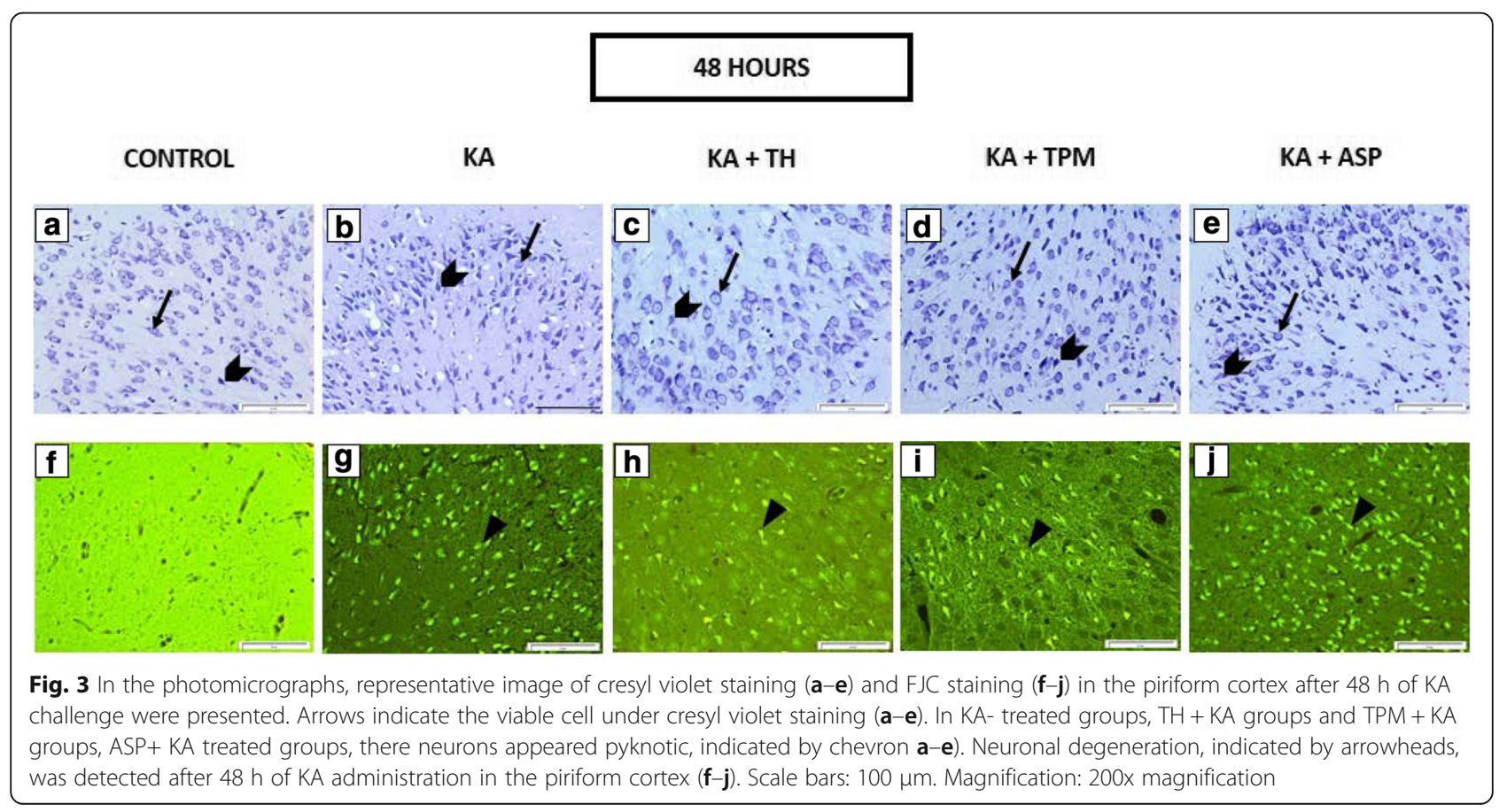


Table 5 Effect of KA-induced excitotoxicity on the number of viable cells in the piriform cortex

\begin{tabular}{|c|c|c|c|c|c|c|c|}
\hline \multirow[t]{2}{*}{ Subgroups } & \multicolumn{5}{|c|}{$\begin{array}{l}\text { Viable cells } \\
\text { Median (lqR) }(n=6)\end{array}$} & \multicolumn{2}{|c|}{ Kruskal-Wallis test $(N=30)$} \\
\hline & CONTROL & $\mathrm{KA}$ & $\mathrm{TH}+\mathrm{KA}$ & $\mathrm{TPM}+\mathrm{KA}$ & $\mathrm{ASP}+\mathrm{KA}$ & X2 stat $(\mathrm{df})$ & $p$ value \\
\hline$\overline{2 \mathrm{H}}$ & $279.17(25.33)$ & $135.17(53.75)^{a}$ & $202.00(38.42)^{a, b}$ & $158.00(3.41)^{a, c}$ & $133.00(27.75)^{\mathrm{a}, \mathrm{c}}$ & $24.46(4)$ & 0.0001 \\
\hline $24 \mathrm{H}$ & $271.83(22.24)$ & $110.50(31.25)^{a}$ & $153.83(57.00)^{\mathrm{a}, \mathrm{b}}$ & $129.00(9.00)^{\mathrm{a}, \mathrm{c}}$ & $90.34(24.25)^{\mathrm{a}, \mathrm{b}, \mathrm{c}}$ & $25.96(4)$ & 0.0000 \\
\hline $48 \mathrm{H}$ & $251.00(40.50)$ & $127.67(54.42)^{a}$ & $160.84(23.42)^{a}$ & $141.00(14.41)^{\mathrm{a}, \mathrm{c}}$ & $80.00(10.17)^{a, b, c}$ & $24.58(4)$ & 0.0001 \\
\hline
\end{tabular}

The results were expressed as the median (IqR). The significant difference was determined by non-parametric test, Kruskal-Wallis test followed by Mann-Whitney $\mathrm{U}$ post-hoc test with $p<0.05$ indicates statistically significant difference. ${ }^{a} p<0.05$ versus control group (MW); ${ }^{b} p<0.05$ versus KA group (MW); ${ }^{c} p<0.05$ versus KA $+\mathrm{TH}$ group (MW)

$24 \mathrm{~h}$ of KA administration, except between KA-treated groups and KA-induced groups pre-treated with $\mathrm{TH}$ after $48 \mathrm{~h}$ of KA administration (Table 5). Therefore, TH pre-treatment significantly attenuated a decrease in the number of viable cells induced by KA.

In addition, there was a significant difference $(p<0.05)$ in the number of viable cells between KA-induced groups pre-treated with $\mathrm{TH}$ and KA-induced groups pre-treated with ASP, and between KA-induced groups pre-treated with $\mathrm{TH}$ and KA-induced groups pre-treated with TPM after $2 \mathrm{~h}, 24 \mathrm{~h}$ and $48 \mathrm{~h}$ of KA administration (Table 5). This indicated that pretreatment with $\mathrm{TH}$ showed a more protective effect than ASP + KA-treated and TPM + KA-treated groups.

\section{Effect of KA-induced excitotoxicity on the number of FJC-positive cells in the piriform cortex}

In the present study, the degenerated neurons were labelled using FJC staining. Histological observation of the FJC-stained slides revealed that FJC-positive cells were absent in all control groups but were identified in all treatments groups that received KA, except in $2 \mathrm{~h}$ treated group, as shown in Fig. 1(f) -(j). The FJC-stained neurons, which emitted bright green fluorescence, could be identified clearly in the $24 \mathrm{~h}$ and $48 \mathrm{~h}$ post-KA administration, as shown in Fig. 2(f) - (j) and Fig. 3(f) -(j).

Statistical analysis of the number of number of FJCpositive cells in the piriform cortex indicated that there was a significant difference $(p<0.05)$ in the number of FJC-positive cells between the groups, for both of $24 \mathrm{~h}$ [X2 $(4)=26.17,(N=30), p=0.0000]$ and
$48 \mathrm{~h}[\mathrm{X} 2(4)=24.71,(N=30), p=0.0001]$ of $\mathrm{KA}$ administration (Table 6). As predicted, KA administration inflicted neuronal degeneration in the piriform cortex of KA-treated groups at $24 \mathrm{~h}$ and $48 \mathrm{~h}$ after $\mathrm{KA}$ administration, which was not the case in the control, where there was no FJC -positive cells observed [see Fig. 1(f) -(j), Fig. 2(f) - (j) and Fig. 3(f) -(j)].

Further examination of the data revealed that there were significant differences $(p<0.05)$ in the number of FJC-positive cells between KA- treated groups and KAtreated groups that received pretreatment of TH, ASP and TPM at $24 \mathrm{~h}$ of KA administration (Table 6). The number of FJC-positive cells in the piriform cortex of $24 \mathrm{~h}$ of KA administration were reduced by pretreatment with TH, TPM and ASP. This indicated that pretreatment with TH, ASP and TPM did have some protective effect in KA-induced neuronal degeneration in the piriform cortex after $24 \mathrm{~h} \mathrm{KA}$ administration.

At $48 \mathrm{~h}$ of KA administration, the quantitative analysis of FJC-positive cells showed that there was a significant difference $(p<0.05)$ between KA-treated groups and KA-induced groups pre-treated with TH and TPM but there was no significance difference $(p>0.05)$ between KA-treated and KA-induced groups pre-treated with ASP (Table 6). This suggested that TH and TPM pretreatment ameliorated the KA-induced neuronal degeneration in the piriform cortex of $48 \mathrm{~h}$ of KA administration but not the case in the pretreatment with ASP.

In addition, quantitative analysis of neuronal degeneration using FJC staining revealed there was a significant

Table 6 Effect of KA-induced excitotoxicity on the number of FJC-positive cells in the piriform cortex

\begin{tabular}{|c|c|c|c|c|c|c|c|}
\hline \multirow[t]{2}{*}{ Subgroups } & \multicolumn{5}{|c|}{$\begin{array}{l}\text { FJC-positive cells } \\
\text { Median (lqR) }(n=6)\end{array}$} & \multicolumn{2}{|c|}{ Kruskal-Wallis test $(N=30)$} \\
\hline & CONTROL & KA & $\mathrm{TH}+\mathrm{KA}$ & $\mathrm{TPM}+\mathrm{KA}$ & $\mathrm{ASP}+\mathrm{KA}$ & $x^{2}$ stat $(d f)$ & $p$ value \\
\hline $24 \mathrm{H}$ & 0 & $225.50(54.25)^{a}$ & $101.67(30.08)^{a, b}$ & $151.33(55.34)^{a, b, c}$ & $161.50(53.92)^{a, b, c}$ & $26.17(4)$ & 0.0000 \\
\hline $48 \mathrm{H}$ & 0 & $168.67(18.00)^{a}$ & $94.00(7.16)^{a, b}$ & $136.67(40.50)^{a, b, c}$ & $168.84(32.66)^{a, c}$ & $24.71(4)$ & 0.0001 \\
\hline
\end{tabular}

The results were expressed as the median (IqR). The significant difference was determined by non-parametric test, Kruskal-Wallis test followed by Mann-Whitney $U$ post-hoc test with $p<0.05$ indicates statistically significant difference. ${ }^{\mathrm{a}} p<0.05$ versus control group (MW); ${ }^{\mathrm{b}} p<0.05$ versus KA group (MW); ${ }^{c} p<0.05$ versus $\mathrm{KA}+$ TH group (MW) 
difference $(p<0.05)$ between KA-induced groups pretreated with $\mathrm{TH}$ and KA-induced groups pre-treated with ASP and between KA-induced groups pre-treated with $\mathrm{TH}$ and KA-induced groups pre-treated with TPM of $24 \mathrm{~h}$ and $48 \mathrm{~h}$ of KA administration. This indicated that pretreatment with $\mathrm{TH}$ showed more protective effect than ASP + KA-treated and TPM + KA-treated groups.

\section{Discussion}

In this experiment, several parameters such as behavioral changes, oxidative stress related markers, and neuronal degeneration were examined to confirm the protective effect of $\mathrm{TH}$ on KA-induced excitotoxicity in a rat model. The administration of KA has been shown to induce a sequence of well-characterized seizures syndrome [2, 7]. Overactivation of glutamate receptors by KA has resulted in the increased production of ROS, which are mediators of oxidative stress. Oxidative stress has been suggested to play an important role in the mechanism of excitotoxicity and neurodegeneration on the different brain regions. Oxidative stress can cause cellular damage and production of ROS, which oxidizes membrane lipids, protein and DNA. There are growing evidences suggesting that oxidative stress has been implicated in the mechanism of excitotoxicity and neurodegeneration on different brain regions after the induction of KA on rodents [5, 6]. Brain is considered to be very vulnerable to oxidative stress because of its great consumption of energy, oxygen and glucose, large amount of peroxidizable polyunsaturated fatty acids and relatively low antioxidant capability.

In the present study, we have demonstrated that administration of KA ( $15 \mathrm{mg} / \mathrm{kg}$ body weight; s.c.) induced epileptic seizures. All KA- treated groups and KAtreated groups that received pretreatment of TH, ASP and TPM had a status epilepticus. In the OFT, KAtreated group has an increased in the locomotor activity (based on number of line crossing) and hyperactivity. Next, we examined TBARS levels and TAS levels in the cerebral cortex after $2 \mathrm{~h}, 24 \mathrm{~h}$ and $48 \mathrm{~h}$ of KA administration. These parameters were associated with oxidative neuronal damage. TBARS levels were elevated whereas, TAS levels was decreased in KA-treated groups after $2 \mathrm{~h}$, $24 \mathrm{~h}$ and $48 \mathrm{~h}$ of KA administration, as compared to control group. In addition, KA-induced epileptic seizure caused neuronal degeneration in the piriform cortex. Our result showed that degenerated neurons were not detected after $2 \mathrm{~h}$ but after $24 \mathrm{~h}$ and $48 \mathrm{~h}$ of KA administration in the piriform cortex.

Previous reports have shown that lipid peroxidation was enhanced by KA in the piriform cortex after $8 \mathrm{~h}$ and $16 \mathrm{~h}$, continued to increase and gradually decreased after $48 \mathrm{~h}$ and 5 days $[6,21,32]$. The elevated TBARS levels after $2 \mathrm{~h}$ of KA administration suggest an increased lipid peroxidation during an early phase of the excitotoxic damage. Sarkar et al. [33], Riba-Bosch and Pérez-Clausell [34] and Hopkins et al. [35] have reported that degenerating neurons in the piriform cortex could be detected after $4 \mathrm{~h}, 6 \mathrm{~h}$ and $8 \mathrm{~h}$ of KA administration and degenerating neurons continued to increase in number throughout the affected areas, reaching its peak at 2 weeks and gradually decreased by 2 months of KA administration. This indicated that neurons in the piriform cortex were undergoing degeneration as early as $4 \mathrm{~h}$ of KA administration and there was a temporal progression of KAinduced neurodegeneration as its continue to increase to its peak and gradually decrease in the number as time goes by after KA administration. Since an increase in TBARS level and a decrease in TAS level occurred as early as after $2 \mathrm{~h}$ of KA administration, this study suggests that lipid is exposed to oxidative damage and strongly indicates the oxidative stress as a possible mechanism of neurodegeneration. Our results demonstrated that oxidative stress was enhanced by KA in the cerebral cortex after $2 \mathrm{~h}, 24 \mathrm{~h}$ and $48 \mathrm{~h}$ of $\mathrm{KA}$ administration.

In this study, we have demonstrated that pretreatment of $\mathrm{TH}$ (at dose of $1.0 \mathrm{~g} / \mathrm{kg}$ body weight) could reduce KA-induced neuronal degeneration in the piriform cortex but had failed to prevent seizures induced by KA. In addition, the increased locomotor activity in the OFT was attenuated by $\mathrm{TH}$ pre-treatment. $\mathrm{TH}$ pre-treatment also significantly reduced the oxidative stress, as shown by an increase in TBARS level and a decrease in TAS level. TH has been reported to contain many bioactive compounds. Several studies have been conducted to investigate the protective effects of specific bioactive compound contained in TH against KA model of excitotoxicity. For example, gallic acid, which is one of the bioactive compound found in $\mathrm{TH}$, has been shown to attenuate the severity of seizure behaviors, reduced neuronal apoptosis and reduced oxidative stress in KAinduced excitotoxicity model [36]. Another bioactive compound is caffeic acid. It has also been shown to provide protection on memory impairment and on oxidative stress induced by KA [37]. These indicate that several important compounds found in TH may play protective roles against KA-induced neuronal degeneration, which may act through their antioxidant activity. Other study on propolis, which is another beehive product, appeared to be effective in preventing KA-induced neuronal loss in the regions of the hippocampus [11]. In addition, propolis was found to attenuate KA-induced seizures and KA-induced oxidative stress in the brain thought to be due to its antioxidant property $[11,38]$. These studies suggested that these antioxidants provide protection against KA-induced neuronal degeneration and seizures 
through antioxidant mechanism. Taken together, the beneficial of $\mathrm{TH}$ may be due to its antioxidant property which is attributed to the presence of flavonoids and phenolic compounds $[12,13]$.

This study also determined the significance of a blockade of the COX pathway and the modulatory effect on KARs, by comparing pretreatment between TH and ASP and between TH and TPM in order to extend our understanding of the pharmacological mechanism of $\mathrm{TH}$. Pretreatment with TH helped to reduce the KA-induced behavioral change, oxidative stress and neuronal degeneration and it showed a more protective effect than ASP + KA-treated and TPM + KA-treated groups. Other study demonstrated that ASP alone did not have any effect on the KA-induced neurodegeneration, while combination of ASP and esculetin, an inhibitor of lipoxygenase (LOX), prevented KA-induced neuronal death [20]. In addition, the findings from Baran et al. [39] and Minutoli et al. [40] studies have suggested that the protection against KAinduced neurodegeneration via combination of COX and LOX inhibitor pathways or via dual inhibitor of COX and LOX pathways were more effective than using only either a COX inhibitor or a LOX inhibitor alone. Administration of KA has caused the activation of phospholipase A followed by an increase level of arachidonic acid [41]. Arachidonic acid then undergoes degradation by $\mathrm{COX}$ or LOX, which form prostaglandins and leukotriene, respectively. The free radicals are formed from prostaglandins synthesis and studies have demonstrated the formation of free radicals due to the action of KA [6, 42]. With the dual inhibition of COX/LOX, the effect of KA-induced excitotoxicity could be attenuated, which blocked both leukotriene and prostaglandins production. This augments its synergistic protective effect, directly through the antiinflammatory mechanisms and indirectly through the anti-radical activity. This may well indicate that blocking of both COX and LOX pathways are necessary to protect the brain against KA-induced seizures and KA-induced neurotoxicity. Furthermore, studies have demonstrated that topiramate has some protective effect against KAinduced seizures and KA-induced hippocampal neurotoxicity $[21,43]$. The discrepancy between the present and previous studies might probably due to differences in experimental conditions such as administration route of KA, administration route of drugs, animal age (young, middleaged or aged), animal strain and animal species (rat or mice).

\section{Conclusion}

Administration of KA (at a dose of $15 \mathrm{mg} / \mathrm{kg}$ body weight; s.c.) induced seizures and resulted in an increase in locomotor activity in KA-treated animals. An increased lipid peroxidation and a decreased total antioxidant status suggestive of an oxidative stress were observed during an early phase of the excitotoxic damage that preceded neurodegeneration. This study suggests that oxidative stress could be a possible mechanism of neurodegeneration and $\mathrm{TH}$ attenuates KAinduced oxidative stress. Hence, TH reduces neurodegeneration in the rat cerebral cortex after $2 \mathrm{~h}, 24 \mathrm{~h}$ and $48 \mathrm{~h}$ of $\mathrm{KA}$ administration due to its antioxidant property.

\section{Additional file}

Additional file 1: The ARRIVE Guidelines Checklist. (DOCX 661 kb)

\section{Abbreviations}

ASP: Aspirin; COX: Cyclooxygenase; FJC: Fluoro Jade C; lqR: Interquartile range; KA: Kainic acid; LOX: Lipoxygenase; OFT: Open field test; ROS: Reactive oxygen species; s.c.: Subcutaneous injection; TAS: Total antioxidant status; TBARS: Thiobarbituric acid reactive substances; TH: Tualang honey;

TPM: Topiramate

\section{Acknowledgements}

The authors would like to express their gratitute to Universiti Sains Malaysia for the grant awarded and also for financial assistance, USM Global Fellowship to the first author, Nur Shafika Mohd Sairazi.

\section{Funding}

This study was funded by Research University Grant (No: 1001/PPSP/811203) from Universiti Sains Malaysia.

\section{Availability of data and materials}

The data supporting the conclusions of this article are included within the article.

\section{Authors' contributions}

NSMS, KNSS, MAA, MS, MM and SAM involved in the experimental design and progress supervision. NSMS carried out the experiments, collected the data and analyzed the data. NSMS wrote the manuscript under supervision of KNSS, MAA, MS, MM and SAM; all authors read and approved the final manuscript.

\section{Competing interests}

The authors declare that they have no competing interest.

Consents for publication

Not applicable.

\section{Ethics approval}

The experimental procedures for animal studies were reviewed and approved by the Animal Ethic Committee of Universiti Sains Malaysia (USM) [USM/Animal Ethics Approval/2011/(68) (305)]. All procedures were conducted according to guidelines approved by the Animal Ethic Committee of USM.

\section{Author details}

${ }^{1}$ Department of Chemical Pathology, School of Medical Sciences, Universiti Sains Malaysia, Health Campus, 16150 Kubang Kerian, Kota Bharu, Kelantan, Malaysia. ${ }^{2}$ Department of Anatomy, School of Medical Sciences, Universiti Sains Malaysia, Health Campus, 16150 Kubang Kerian, Kota Bharu, Kelantan, Malaysia. ${ }^{3}$ Department of Neurosciences, School of Medical Sciences, Universiti Sains Malaysia, Health Campus, 16150 Kubang Kerian, Kota Bharu, Kelantan, Malaysia. ${ }^{4}$ Department of Pharmacology, School of Medical Sciences, Universiti Sains Malaysia, Health Campus, 16150 Kubang Kerian, Kota Bharu, Kelantan, Malaysia. 


\section{References}

1. Doble A. The Role of Excitotoxicity in Neurodegenerative Disease: Implications for Therapy. Pharmacol Ther. 1999;81(3):163-221.

2. Zhang X, Gelowitz DL, Lai CT, Boulton AA, Yu PH. Gradation of kainic acidinduced rat limbic seizures and expression of hippocampal heat shock protein-70. Eur J Neurosci. 1997;9(4):760-9.

3. Zeng LH, Zhang HD, Xu CJ, Bian YJ, Xu XJ, Xie QM, Zhang RH. Neuroprotective effects of flavonoids extracted from licorice on kainateinduced seizure in mice through their antioxidant properties. J Zhejiang Univ Sci B. 2013;14(11):1004-12.

4. Golechha M, Chaudhry U, Bhatia J, Saluja D, Arya DS. Naringin protects against kainic acid-induced status epilepticus in rats: evidence for an antioxidant, anti-inflammatory and neuroprotective intervention. Biol Pharm Bull. 2011;34(3):360-5.

5. Gluck MR, Jayatilleke E, Shaw S, Rowan AJ, Haroutunian V. CNS oxidative stress associated with the kainic acid rodent model of experimental epilepsy. Epilepsy Res. 2000;39(1):63-71.

6. Bruce AJ, Baudry M. Oxygen free radicals in rat limbic structures after kainate-induced seizures. Free Radic Biol Med. 1995;18(6):993-1002.

7. Ben-Ari Y. Limbic seizure and brain damage produced by kainic acid: Mechanisms and relevance to human temporal lobe epilepsy. Neuroscience. 1985;14(2):375-403.

8. Si PP, Zhen JL, Cai YL, Wang WJ, Wang WP. Salidroside protects against kainic acid-induced status epilepticus via suppressing oxidative stress. Neurosci Lett. 2016:618:19-24.

9. Jeong KH, Jung UJ, Kim SR: Naringin Attenuates Autophagic Stress and Neuroinflammation in Kainic Acid-Treated Hippocampus In Vivo. Evid-Based Complement Alternat Med 2015:9.

10. Gupta YK, Briyal S, Sharma M. Protective effect of curcumin against kainic acid induced seizures and oxidative stress in rats. Indian J Physiol Pharmacol. 2009;53(1):39-46.

11. Kwon YS, Park DH, Shin EJ, Kwon MS, Ko KH, Kim WK, Jhoo JH, Jhoo WK Wie MB, Jung BD, et al. Antioxidant propolis attenuates kainate-induced neurotoxicity via adenosine $\mathrm{A} 1$ receptor modulation in the rat. Neurosci Lett. 2004;355(3):231-5.

12. Chua LS, Rahaman NLA, Adnan NA, Eddie Tan T. Antioxidant Activity of Three Honey Samples in relation with Their Biochemical Components. J Anal Methods Chem. 2013;2013:8

13. Khalil M, Alam N, Moniruzzaman M, Sulaiman SA, Gan SH. Phenolic Acid Composition and Antioxidant Properties of Malaysian Honeys. J Food Sci. 2011;76(6):C921-8

14. Erejuwa OO, Gurtu S, Sulaiman SA, Ab Wahab MS, Sirajudeen KNS, Salleh MS. Hypoglycemic and antioxidant effects of honey supplementation in streptozotocin-induced diabetic rats. Int J Vitam Nutr Res. 2010;80(1):74-82

15. Zaid SS, Sulaiman SA, Sirajudeen KNS, Othman NH. The effects of Tualang honey on female reproductive organs, tibia bone and hormonal profile in ovariectomised rats-animal model for menopause. BMC Complement Altern Med. 2010;10:82

16. Saxena AK, Phyu HP, Al-Ani IM, Oothuman P. Improved spatial learning and memory performance following Tualang honey treatment during cerebral hypoperfusion-induced neurodegeneration. J Transl Sci. 2016;2(5):264-71.

17. Saxena AK, Phyu HP, Al-Ani IM, Talib NA. Potential protective effect of honey against chronic cerebral hypoperfusion-induced neurodegeneration in rats. J Anat Soc India. 2014;63(2):151-5.

18. Kamarulzaidi MA. Mohd. Yusoff MYZ, Mohamed AM, Hasan Adli DS: Tualang Honey Consumption Enhanced Hippocampal Pyramidal Count and Spatial Memory Performance of Adult Male Rats. Sains Malaysiana. 2016:45(2):215-20.

19. Al-Rahbi B, Zakaria R, Othman Z, Hassan A, Mohd Ismail ZI, Muthuraju S. Tualang honey supplement improves memory performance and hippocampal morphology in stressed ovariectomized rats. Acta Histochem. 2014;116(1):79-88.

20. Kim HC, Jhoo WK, Bing G, Shin EJ, Wie MB, Kim WK, Ko KH. Phenidone prevents kainate-induced neurotoxicity via antioxidant mechanisms. Brain Res. 2000;874(1):15-23.

21. Kubera M, Budziszewska B, Jaworska-Feil L, Basta-Kaim A, Leskiewicz M, Tetich M, Maes M, Kenis G, Marciniak A, Czuczwar SJ, et al. Effect of topiramate on the kainate-induced status epilepticus, lipid peroxidation and immunoreactivity of rats. Pol J Pharmacol. 2004:56(5):553-61.

22. Kilkenny C, Browne WJ, Cuthill IC, Emerson M, Altman DG. Improving bioscience research reporting: the ARRIVE guidelines for reporting animal research. PLoS Biol. 2010;8(6):e1000412.
23. Wang P, Ren RN, Cai SY, Chen XM. Ye LY: [Neuroprotective effects of topiramate and folic acid on young rats with kindling-induced epilepsy]. Zhongguo Dang Dai Er Ke Za Zhi. 2008;10(1):65-9.

24. Sobaniec-Lotowska ME, Lotowska JM. The neuroprotective effect of topiramate on the ultrastructure of pyramidal neurons of the hippocampal CA1 and CA3 sectors in an experimental model of febrile seizures in rats. Folia Neuropathol. 2011;49(3):230-6.

25. Chen J, Quan QY, Yang F, Wang Y, Wang JC, Zhao G, Jiang W. Effects of lamotrigine and topiramate on hippocampal neurogenesis in experimental temporal-lobe epilepsy. Brain Res. 2010;1313:270-82.

26. Milatovic D, Gupta RC, Dettbarn W-D. Involvement of nitric oxide in kainic acid-induced excitotoxicity in rat brain. Brain Res. 2002;957(2):330-7.

27. Drexel M, Preidt AP, Sperk G. Sequel of spontaneous seizures after kainic acid-induced status epilepticus and associated neuropathological changes in the subiculum and entorhinal cortex. Neuropharmacology. 2012;63(5):806-17.

28. Paxinos G, Watson C. The Rat Brain in Stereotaxic Coordinates. 6th ed. New York: Academic; 2007.

29. Ohkawa H, Ohishi N, Yagi K. Assay for lipid peroxides in animal tissues by thiobarbituric acid reaction. Anal Biochem. 1979;95:351-8.

30. Koracevic D, Koracevic G, Djordjevic V, Andrejevic S, Cosic V. Method for the measurement of antioxidant activity in human fluids. J Clin Pathol. 2001:54(5):356-61.

31. Schmued LC, Stowers CC, Scallet AC, Xu L. Fluoro-Jade C results in ultra high resolution and contrast labeling of degenerating neurons. Brain Res. 2005;1035(1):24-31.

32. Swamy M, Yusof WRW, Sirajudeen K, Mustapha Z, Govindasamy C. Decreased glutamine synthetase, increased citrulline-nitric oxide cycle activities, and oxidative stress in different regions of brain in epilepsy rat model. J Physiol Biochem. 2011;67(1):105-13.

33. Sarkar S, Raymick J, Schmued L. Temporal progression of kainic acid induced changes in vascular laminin expression in rat brain with neuronal and glial correlates. Curr Neurovasc Res. 2012;9(2):110-9.

34. Riba-Bosch À, Pérez-Clausell J. Response to kainic acid injections: changes in staining for zinc, FOS, cell death and glial response in the rat forebrain. Neuroscience. 2004;125(3):803-18.

35. Hopkins KJ, Wang G-J, Schmued LC. Temporal progression of kainic acid induced neuronal and myelin degeneration in the rat forebrain. Brain Res. 2000;864(1):69-80

36. Huang HL, Lin CC, Jeng KC, Yao PW, Chuang LT, Kuo SL, Hou CW. Fresh green tea and gallic acid ameliorate oxidative stress in kainic acid-induced status epilepticus. J Agric Food Chem. 2012;60(9):2328-36.

37. Kumar A, Prakash A, Pahwa D. Galantamine potentiates the protective effect of rofecoxib and caffeic acid against intrahippocampal Kainic acid-induced cognitive dysfunction in rat. Brain Res Bull. 2011;85(3-4):158-68.

38. Swamy M, Norlina W, Azman W, Suhaili D, Sirajudeen KN, Mustapha Z, Govindasamy C. Restoration of glutamine synthetase activity, nitric oxide levels and amelioration of oxidative stress by propolis in kainic acid mediated excitotoxicity. Afr J Tradit Complement Altern Med. 2014;11(2):458-63.

39. Baran H, Vass K, Lassmann H, Hornykiewicz O. The cyclooxygenase and lipoxygenase inhibitor BW755C protects rats against kainic acid-induced seizures and neurotoxicity. Brain Res. 1994;646(2):201-6.

40. Minutoli L, Marini H, Rinaldi M, Bitto A, Irrera N, Pizzino G, Pallio G, Calò M, Adamo E, Trichilo V, et al. A Dual Inhibitor of Cyclooxygenase and 5-Lipoxygenase Protects Against Kainic Acid-Induced Brain Injury. Neuromolecular Med. 2015;17(2):192-201.

41. Farooqui AA, Ong WY, Horrocks LA. Neuroprotection abilities of cytosolic phospholipase A2 inhibitors in kainic acid-induced neurodegeneration. Curr Drug Targets Cardiovasc Haematol Disord. 2004;4(1):85-96.

42. Baran $\mathrm{H}$, Heldt $\mathrm{R}$, Hertting $\mathrm{G}$. Increased prostaglandin formation in rat brain following systemic application of kainic acid. Brain Res. 1987;404(1-2):107-12.

43. Park HJ, Kim HJ, Park HJ, Ra J, Zheng LT, Yim SV, Chung JH. Protective effect of topiramate on kainic acid-induced cell death in mice hippocampus. Epilepsia. 2008;49(1):163-7. 